\title{
Non-Protocol Disposition Event
}

National Cancer Institute

\section{Source}

National Cancer Institute. Non-Protocol Disposition Event. NCI Thesaurus. Code C150824.

The group of incidents that are not driven by the protocol but that may occur during a clinical trial and describe whether a subject completed the study epoch or the reason this event did not occur. 